\title{
(6) \\ OPEN ACCESS \\ Practical advice for avoidance of pain associated with insertion of intrauterine contraceptives
}

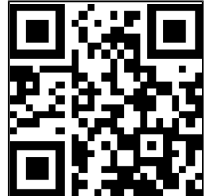

Open Access Scan to free content
${ }^{1}$ Professor of Gynaecology, Department of Obstetrics and Gynaecology, Faculty of Medical Sciences, University of Campinas (UNICAMP), Campinas, SP, Brazil

${ }^{2}$ Consultant in Community Gynaecology and Reproductive Healthcare, Newcastle Hospitals, Community Health, New Croft Centre, Newcastle upon Tyne, UK

${ }^{3}$ Director, Gynmed Clinic, Vienna, Austria and Department of Women's and Children's Health, Karolinska Institutet, Karolinska University Hospital, Stockholm, Sweden

${ }^{4}$ Professor and Associate Chair, Department of Obstetrics and Gynecology, University of Florida College of Medicine-Jacksonville, Jacksonville, FL, USA

${ }^{5}$ Professor of Obstetrics and Gynecology and Department Chair, Department of Women's and Children's Health,

Karolinska Institutet, Karolinska University Hospital, Stockholm, Sweden

\section{Correspondence to}

Dr Luis Bahamondes,

Department of Obstetrics and Gynaecology, Faculty of Medical Sciences, University of Campinas (UNICAMP), Caixa Postal 6181, Campinas, SP 13084-971, Brazil; bahamond@caism. unicamp.br

Received 11 April 2013 Revised 24 June 2013 Accepted 30 July 2013 Published Online First 27 September 2013

To cite: Bahamondes $\mathrm{L}$, Mansour D, Fiala C, et al. J Fam Plann Reprod Health Care 2014:40:54-60.

\author{
Luis Bahamondes, ${ }^{1}$ Diana Mansour, ${ }^{2}$ Christian Fiala, ${ }^{3}$ \\ Andrew M Kaunitz, ${ }^{4}$ Kristina Gemzell-Danielsson ${ }^{5}$
}

\begin{abstract}
Few studies in the scientific literature provide clear direction on the prevention or management of pain associated with intrauterine contraceptive (IUC) placement. Those that have been published have studied small numbers of women and fail to provide definitive conclusions. There are also no guidelines available detailing recognised standard approaches to this problem. The consensus recommendations in this review focus primarily on non-pharmacological and often non-evidencebased interventions. This review includes general considerations, practical recommendations for both routine and more difficult cases and guidance on the optimal choice of instruments. General considerations, including pre-insertion counselling, the setting for the procedure, the confidence and technique of the provider and the interplay between the provider and assistant, can influence women's level of anxiety and, in turn, influence their perception of pain and their overall experience. Further studies are required to refine the optimal strategy for managing pain associated with IUC insertion.
\end{abstract}

\section{INTRODUCTION}

Intrauterine contraceptives (IUCs) are amongst the most effective of all contraceptive methods ${ }^{1}{ }^{2}$ and, according to guidelines, are suitable for most women, regardless of age or parity. ${ }^{3-8}$ Most IUC insertions involve a brief procedure, without complication, regardless of parity. ${ }^{9}$ Although there is evidence that nulliparous women (nulligravidas and those who have been pregnant but never given birth) may experience more placement-related pain than their parous counterparts, ${ }^{10-12}$ the majority experience a level of pain that is similar in intensity to that of menstruation. ${ }^{13}$ However, a proportion of both nulliparous and parous women experience substantial pain: in a study of nulliparous women, approximately 17\% reported severe pain associated with placement of a levonorgestrel-releasing intrauterine system (LNG IUS). ${ }^{14}$ In a study of mainly multiparous women (mean of two prior births; $n=46$ ), approximately $11 \%$ reported severe insertion-related pain. ${ }^{15}$ Such individuals need effective pain relief.

There are few studies in the scientific literature that give clear guidance on the management of pain associated with IUC fitting. Those that have been published have studied small numbers of women and fail to provide definitive conclusions. There are also no guidelines available detailing recognised standard approaches to this problem. In addition, data are lacking on the clinical benefits of pain management for IUC placements, and no standard has been established.

Pharmacological strategies used by some health care providers (HCPs) include treatment given before the procedure (cervical priming, oral analgesia and local anaesthesia); during the procedure (local anaesthesia administered reactively); and after the procedure [non-steroidal anti-inflammatory drugs (NSAIDs) and opioid analgesia]. However, of the preventative interventions that have been adequately evaluated, none has shown clear evidence that pain is significantly reduced during or after routine IUC placement. ${ }^{16}$ In addition, most of the regimens evaluated (e.g. those for cervical priming, and pre-placement oral analgesia) have been adopted from their use in other gynaecological procedures such as hysteroscopy or surgical abortion. ${ }^{17}$

Overall, the published evidence indicates the need for effective pain relief for some women undergoing IUC placement, but offers limited guidance in this regard. This review therefore presents consensus 
recommendations for improving women's experiences of IUC placement, with a specific focus on pain relief.

\section{METHODOLOGY FOR DEVELOPING CONSENSUS RECOMMENDATIONS}

The authors were invited to a 1-day meeting to share their practical experience when fitting IUCs. This was supported by an educational grant from Bayer plc, manufacturer of the LNG IUS (Mirena ${ }^{\circledR}$ ) and a copper-releasing intrauterine device (IUD, Nova $\mathrm{T}^{\circledR}$ ). The participants reviewed published evidence, shared their personal experiences and produced consensus recommendations for pain management in IUC placement.

The present authors have recently published a review of the evidence covering pain management strategies for IUC insertion. ${ }^{18}$ The review presents evidence-based recommendations and points out gaps in the data and areas for further research. A total of 17 studies including 12 randomised controlled trials (RCTs) provided no conclusive evidence that any prophylactic pharmacological intervention reduces pain associated with IUC insertion; however, one review reported that NSAIDs may be effective in treating post-insertion pain.

In the absence of clear evidence supporting pharmacological interventions, the scope of the discussions was expanded to include various non-pharmacological, non-evidence-based strategies used in clinical practice. The authors shared strategies from their own practice and, through debate, areas of consensus were identified. Attention to such factors may help to improve a woman's experience of IUC placement and these are detailed in the following sections.

\section{GENERAL RECOMMENDATIONS FOR IMPROVING WOMEN'S EXPERIENCE}

\section{Pre-placement counselling}

Women's anxiety has been shown to contribute to higher levels of perceived pain during IUC placement. Women who believe that IUC placement will be a painful procedure are more anxious and probably more likely to take oral analgesics before the placement; consequently, reduction of anxiety is a good strategy to reduce pain during IUC placement. ${ }^{19}$ Non-pharmacological interventions, such as pre-placement counselling, 'verbal anaesthesia' and distraction during the procedure may, therefore, be effective at reducing pain, although no studies on these approaches have been published to date.

Counselling materials should be available that provide the woman with realistic information about what to expect during and after the procedure. The level of pain that women experience is highly variable, making it difficult to predict the level of discomfort that an individual is likely to experience; this should be explained in counselling. However, it can be useful to explain the measures that will be taken to minimise discomfort. The authors support the provision of a sympathetic person to provide a 'vocal local' when fitting IUCs.

\section{The clinical setting}

The placement of an IUC is a minor, office-based procedure and does not require the formal setting of an operating room or special treatment room. A bimanual examination must be performed prior to the fitting of an IUC to help exclude pelvic pathology and to determine the uterine position to reduce the risk of uterine perforation. The authors suggest that ideally the clinic room should be furnished with a gynaecology couch with leg rests. If only a conventional flat couch is available, the woman should be positioned so that the HCP can fit the IUC from the end of the couch. This will facilitate visualisation of the cervix, cause less discomfort for the HCP and may improve the ease of the insertion. However, the sensitivities and expectations of the woman should always be taken into account. The need for cervical dilatation or pain relief is difficult to predict. We therefore recommend that the necessary equipment for provision of local anaesthesia and for cervical dilatation is kept immediately to hand.

Vasovagal reactions can be difficult to predict; accordingly, many HCPs keep the necessary drugs and equipment to deal with these immediately accessible during the procedure. These reactions are more likely to occur in difficult or non-routine cases and during procedures performed by less experienced HCPs, who may have less technical expertise or confidence. Recommendations for emergency drugs and equipment vary between countries and providers should follow their own local guidelines.

\section{Confidence and technique of the HCP}

IUC placements should ideally be performed by experienced HCPs who have been appropriately trained and who have maintained their skills. HCPs in training must carry out placements under supervision until both the trainee and trainer are satisfied that clinical competence has been achieved. HCPs should audit their practice and, if appropriate, consider referring complicated cases to a more experienced colleague. The number of placements performed per year is important with regard to outcome. ${ }^{20} 21$

\section{Team interplay between the assistant and HCP}

Women notice the team interplay between the assistant and the HCP; a well-functioning team inspires confidence and reduces women's anxiety, which may, in turn, have a positive impact on their perception of pain.

\section{Timing in the menstrual cycle}

IUCs may be fitted at any point in the menstrual cycle with insertion-related pain being similar throughout. However, some HCPs prefer to fit IUCs within the 
first 7 days of the cycle as they feel that women experience less pain (however, with no scientific evidence) and because there is virtual certainty that the woman could not be pregnant.

\section{RECOMMENDATIONS FOR ROUTINE PLACEMENTS}

The IUC package should not be opened until the uterus has been successfully sounded

Difficulty with sounding can indicate a potentially difficult procedure. In this situation, the HCP should ensure that local anaesthesia and fine cervical dilators are available.

\section{Clinicians should be aware of the origins of pain}

During the insertion procedure, HCPs should be aware of actions that could potentially cause pain and should minimise these where possible. An accurate initial bimanual examination will not only help to prevent instrumental or IUC perforation, but may help to reduce insertion pain. For example, if a woman has a retroverted uterus, unnecessary pain will be experienced if the sound is directed anteriorly. Applying a tenaculum or Allis forceps, applying traction to the cervix, stretching or dilating the external or internal cervical os (e.g. when inserting an Os Finder, when passing the sound, dilators if needed, or the IUC insertion tube), and touching the uterine fundus during sounding or when ensuring that the IUC is placed fundally, may all cause discomfort or pain. Potential pain or problems should be anticipated and each step should be explained to the woman to help to minimise her anxiety and discomfort.

\section{Clinicians should be open to women's differing needs for communication during the procedure}

Women vary in the amount of information that they wish to receive during the procedure. Therefore it is important to 'know your patient' and to be aware that women from different cultures respond differently to the placement procedure. The presence of an assistant during the procedure may help to reduce the woman's anxiety.

\section{Routine use of ultrasound is not necessary but it may be used in settings where it is readily available}

The availability of ultrasound is not a prerequisite for placing IUCs. However, when ultrasound is readily available it can be used and it may be helpful in certain situations. For example, after a difficult fitting ultrasound may be useful for confirming that the device has been positioned correctly in the uterine cavity.

\section{Routine cervical dilatation is not necessary}

Routine dilatation of the cervix beyond the diameter of the IUC inserter is not recommended because it results in additional pain as well as increasing the risk of a vasovagal episode. When the IUC inserter is first applied to the cervical os, we recommend that the clinician should pause for a few moments to allow the os to slowly stretch and accommodate the inserter. This makes the placement procedure easier and potentially less painful. Few women, nulligravid or parous, require cervical dilatation for IUC placement. However this may be necessary in some cases even when no particular difficulty has been encountered with sounding. ${ }^{9} 14$

\section{Clinicians should use non-pharmacological pain management strategies}

There is currently no effective evidence-based strategy for managing pain in women who experience severe discomfort during IUC placement. As mentioned previously, 'verbal anaesthesia' provided by an assistant is, in our experience, one of the most effective ways of reducing pain and anxiety. However, in routine practice the use of a small warm-water bottle inside a disposable cover, held by the woman suprapubically, may also be helpful. Concentration on holding the bottle can distract her during the procedure and the warmth can be comforting. Chemical warming packs may be a suitable alternative.

\section{RECOMMENDATIONS FOR MORE DIFFICULT CASES}

Our recommended approaches to various scenarios in which IUC placement might be expected to be more difficult are outlined below.

\section{Scenario 1: Nulliparous and nulligravid women}

IUC insertions for nulliparous and nulligravid women should be considered to be routine cases. Most of these women do not experience severe placementrelated pain. However, placement may be more painful for some of these women when compared with those who have experienced progressive labour. For all IUC candidates, but particularly nulliparous and nulligravid women, the clinician should be prepared to administer a local anaesthetic if the woman experiences moderate or severe pain, if she requests pain relief, or if dilatation of the cervix is required.

\section{Scenario 2: Uterine sounding is difficult and/or painful}

It is difficult to predict whether a woman will have complications or pain associated with IUC insertion. If uterine sounding proves to be difficult and/or painful, we recommend the administration of local anaesthesia as an intracervical or paracervical block before proceeding to cervical dilatation.

\section{Scenario 3: Cervical stenosis}

For cases in which attempted uterine sounding demonstrates stenosis at either the external or internal cervical os, we recommend consideration of administration of local anaesthesia (see later) followed by gentle use of an Os Finder (a malleable plastic tapered 
dilator) or a suitable, preferably tapered, metal dilator. If placement is not possible despite these measures, the procedure should be discontinued.

If an IUC remains the woman's contraceptive method of choice, consideration may be given to cervical priming with misoprostol, administered buccally or vaginally 3 hours before a repeat procedure, or sublingually 1 hour beforehand. However it should be noted that several studies have suggested that while misoprostol priming may permit easier passage of the IUC (see Scenario 3), it is not effective in reducing pain and it may, in fact, increase overall procedural pain, ${ }^{15} 22-24$ often due to uterine cramping.

Although misoprostol has been studied extensively for cervical priming to ease IUC placement, the data are conflicting. One $\mathrm{RCT}^{15}$ showed no benefit when misoprostol was compared to placebo given prior to the removal and replacement of a LNG IUS. Another $\mathrm{RCT}^{22}$ allocated the women to receive either $400 \mu \mathrm{g}$ misoprostol sublingually with $100 \mathrm{mg}$ diclofenac, or $100 \mathrm{mg}$ diclofenac alone. Use of misoprostol was associated with significantly easier IUC insertion. However, in a further $\mathrm{RCT}^{23}$ comparing misoprostol with placebo, placement failure and placement-related complications were similar in both groups. In all three RCTs, reported placement pain was similar in those receiving misoprostol, placebo $^{15}{ }^{23}$ or diclofenac alone. ${ }^{22}$ In the opinion of the authors, if misoprostol is to be used for cervical priming, a non-steroidal analgesic (NSAID) should be offered in addition in order to reduce its prostaglandin-mediated side effects including uterine cramping. ${ }^{18}$

\section{Scenario 4: A woman with extreme anxiety due to anticipation of severe pain with IUC placement}

A woman who anticipates that IUC placement will be associated with severe pain may be extremely anxious and may request that the procedure be performed under general anaesthesia or sedation. Overall, the risks of general anaesthesia are greater than those of local anaesthesia. IUC placement performed under general anaesthesia or sedation may also be associated with a slightly increased risk of uterine perforation due to the use of excessive force at the internal os, although when performed by experienced clinicians, with appropriate dilators or an Os Finder, the risk is still very low. Use of general anaesthesia should, therefore, be limited to special situations, such as when a woman with learning difficulties has opted for IUD placement and it is considered that the procedure would be impossible with no or local anaesthesia. As an alternative to general anaesthesia, conscious sedation following cervical priming could be considered in a setting that should include adequate patient monitoring. In most cases, however, the woman's anxiety can usually be addressed with appropriate counselling before the procedure and reassurance and/or distraction during the procedure. In a review of approximately 1000 IUC placements performed over 1 year in Newcastle upon Tyne, UK, only one case was referred for IUC placement under general anaesthesia (D Mansour, personal communication). Furthermore, in a Brazilian study evaluating LNG IUS placement in nulligravid $(n=159)$ and parous $(n=477)$ women, no anaesthesia was used and insertion failure was observed in only $0.4 \%$ of women. ${ }^{9}$

\section{CERVICAL ANAESTHESIA}

Cervical anaesthesia can be administered paracervically or intracervically. ${ }^{25}$ Expert providers differ in their preferences and, in the absence of a RCT comparing these methods for pain relief during IUC placement, there is little evidence that either method is more effective than the other.

\section{Intracervical lidocaine gel}

Two RCTs evaluated the use of intracervical 2\% lidocaine gel versus placebo prior to uterine sounding. One trial used a cotton swab soaked in the study gel or placebo and inserted into the cervical canal to the level of the internal os for 60 seconds. ${ }^{26}$ No benefit was found for lidocaine gel over placebo as used in these two studies. ${ }^{26} 27$

\section{Paracervical block}

A paracervical block aims for regional anaesthesia of the inferior hypogastric plexus and ganglia. ${ }^{28} \mathrm{~A}$ wide variety of methods and techniques has been published. Traditionally, a paracervical block was administered using a $20 \mathrm{ml}$ syringe, a 21 gauge needle and $15-20 \mathrm{ml}$ $1 \%$ lidocaine, which was injected into the cervicovaginal junction to a depth of 3-7 $\mathrm{mm}$ at two to six injection sites. More recently, the technique for paracervical blockade has been modified. Although there are no studies to support this practice, many clinicians now use a dental syringe with a finer (27 gauge) needle to administer a smaller volume of local anaesthetic, one or two $2.2 \mathrm{ml}$ vials of $1 \%$ lidocaine, $2-3 \%$ mepivacaine or $4 \%$ prilocaine, with or without a vasoconstrictor. A small amount of local anaesthetic is injected into the anterior cervical lip to reduce any discomfort caused by applying the tenaculum. The remainder of the local anaesthetic is then injected into the cervicovaginal junction, either laterally or at the 4 and 8 o'clock positions.

\section{Intracervical block}

An intracervical block is an injection of local anaesthesia into the cervical stroma, similar to the local anaesthetic block given when performing loop excision procedures for treatment of cervical dysplasia. Descriptions vary from injection into the anterior lip of the cervix only, to circumferential injection at four positions around the external os. ${ }^{25}$ The authors suggest injecting the local anaesthetic through the cervical canal, as close as possible to the internal os, at the 4 and 8 o'clock positions. The advantage of 

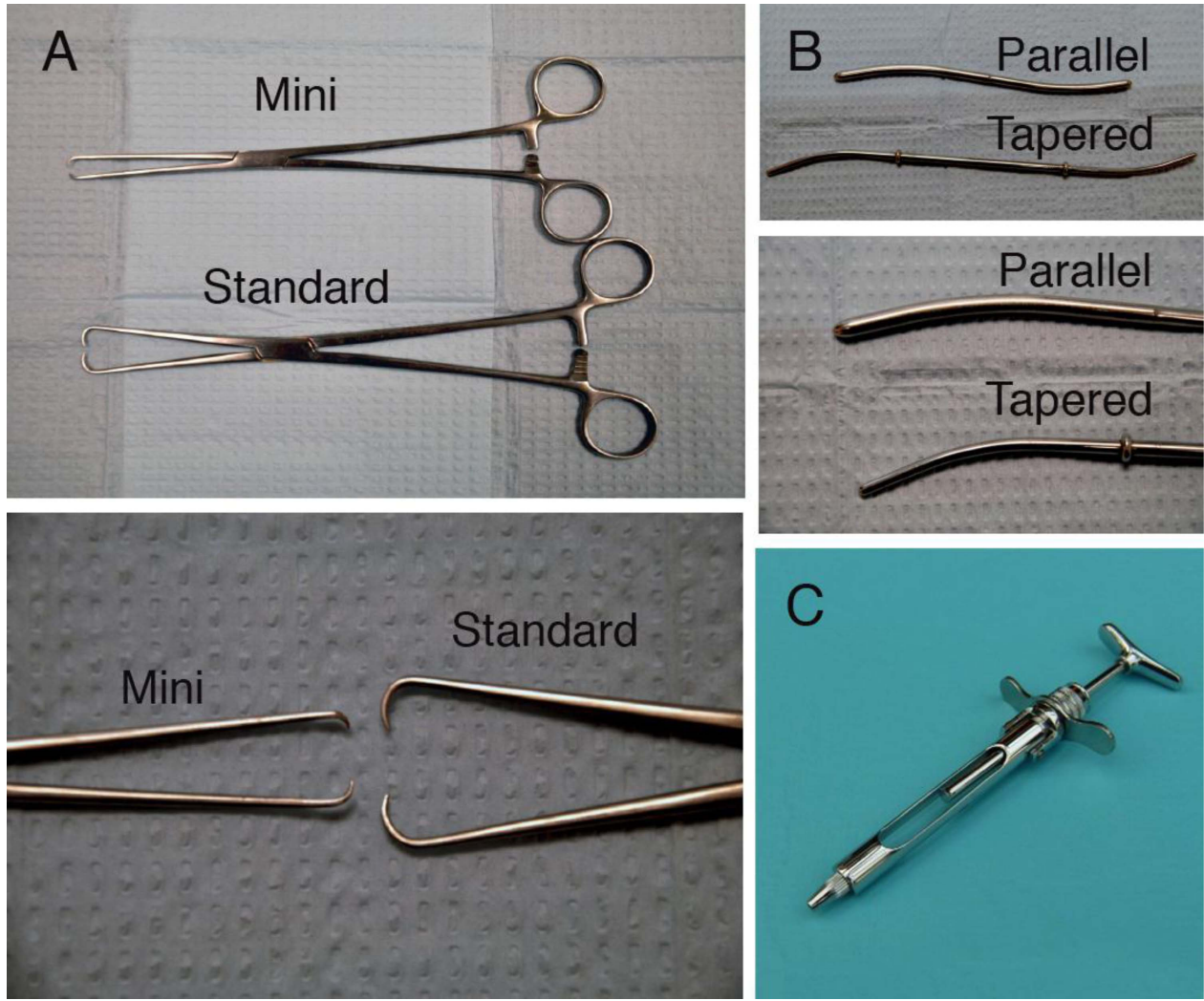

Figure 1 Recommended instruments for performing intrauterine contraceptive placements. (A) Mini and standard tenacula. (B) Tapered versus parallel cervical dilators. (C) Dental syringe.

intracervical block by either method is that when using a dental syringe, only one or occasionally two $2.2 \mathrm{ml}$ vials of local anaesthetic are needed, resulting in a lower injection volume and, therefore, less pain during the injection; there is no risk of intravascular injection; and the onset of the anaesthetic effect is immediate. Prilocaine with a non-adrenaline vasoconstrictor, felypressin, is used to avoid adrenaline-related adverse reactions (such as feeling faint or palpitations) and to reduce bleeding following the injection.

\section{THE MOST APPROPRIATE INSTRUMENTS}

Regardless of whether a placement is considered routine or difficult, using the most appropriate instruments may optimise outcomes and improve women's experience of the procedure. In general, we recommend resterilisable non-disposable instruments (Figure 1). The use of disposable plastic and metal instruments (mainly specula) is being adopted in many settings due to the increasingly rigorous requirements for sterilisation. However, most plastic tenacula fail to grasp the cervix effectively and plastic scissors are not sharp enough or strong enough to cut the IUC threads. Based on our collective experience in fitting IUCs, we recommend the following instruments.

\section{Speculum}

Use of a short bivalve speculum usually facilitates IUC placement. The advantage of a short speculum is that more gentle traction can be placed on the cervix, straightening the cervical canal and easing placement by bringing the cervix closer to the introitus.

\section{Tenaculum}

The authors advise that a tenaculum should always be applied to stabilise the cervix and to reduce the risk of uterine perforation by gently straightening the cervico-uterine angle. Use of a fine tenaculum (sometimes marketed for use in saline infusion sonography) or Judd-Allis forceps provides several advantages: less pain associated with application (one of the most painful aspects of the IUC placement procedure), less trauma to and bleeding from cervical tissue and greater ease in grasping a 'flat cervix'.

\section{Syringe for administration of local anaesthesia}

Administration of a local anaesthetic into the cervix using a $10-20 \mathrm{ml}$ disposable syringe is cumbersome. The use of a dental syringe and needle greatly facilitates the administration of small volumes of more effective local anaesthetic. The design of the dental syringe gives the HCP more control of the syringe and plunger; the syringe is narrow and the cervix can therefore be viewed more easily when administering a paracervical or intracervical block. 


\section{Dilators}

We recommend the use of tapered cervical dilators increasing from 3 to $5 \mathrm{~mm}$ in diameter, in preference to cylindrical (Hegar) dilators. These gently dilate the cervical os and canal. However, if the only dilators available are of the cylindrical type, we recommend gentle sequential use of 3,4 and $5 \mathrm{~mm}$ diameters. Only in exceptional cases should it be necessary to use a $6 \mathrm{~mm}$ diameter dilator. We generally recommend the use of resterilisable instruments. However, Os Finders and plastic tapered dilators are available; these can be resterilisable or disposable and are a good option. We do not recommend the use of osmotic dilators (e.g. laminaria) for cervical priming because of a lack of supporting studies.

\section{CONCLUSIONS}

No preventative pharmacological intervention has been adequately evaluated in an RCT setting and been shown to significantly reduce pain associated with IUC placement. General considerations, including preplacement counselling, the setting in which the actual procedure is performed, the confidence and technique of the clinician and the interplay between the HCP and assistant, can positively or negatively impact on a woman's level of anxiety and therefore potentially influence her perception of pain as well as her overall experience. Ongoing efforts are required to refine the optimal strategy for managing pain associated with IUC placement and to improve women's experience of the procedure. In addition, there will be benefits if best practices are shared internationally.

Acknowledgements The writing of this manuscript was independently led by the authors with the assistance of Sonya Haslam, who provided medical writing support.

Funding Bayer Health funded the medical writing of this study.

Competing interests The authors were invited to meet and discuss the practical management of intrauterine contraceptive fitting by Bayer AG and this was supported by an educational grant. No honoraria were provided to the authors.

Provenance and peer review Not commissioned; externally peer reviewed.

Open access This is an Open Access article distributed in accordance with the Creative Commons Attribution Non Commercial (CC BY-NC 3.0) licence, which permits others to distribute, remix, adapt, build upon this work noncommercially, and license their derivative works on different terms, provided the original work is properly cited and the use is non-commercial. See: http://creativecommons.org/licenses/by$\mathrm{nc} / 3.0 /$

\section{REFERENCES}

1 Trussell J. Contraceptive failure in the United States. Contraception 2011;83:397-404.

2 Winner B, Peipert JF, Zhao Q, et al. Effectiveness of long-acting reversible contraception. N Engl J Med 2012;366:1998-2007.

3 Lyus R, Lohr P, Prager S. Use of the Mirena LNG-IUS and Paragard CuT380A intrauterine devices in nulliparous women. Contraception 2010;81:367-371.
4 National Institute for Health and Care Excellence. Clinical Knowledge Summaries. UK Medical Eligibility Criteria for use of the levonorgestrel-releasing intrauterine system. 2011. http:// www.cks.nhs.uk/contraception_ius_iud/management/scenario_ intrauterine_system/starting_a_levonorgestrel_releasing_ius/uk medical_eligibility_criteria [accessed 26 November 2012].

5 National Institute for Health and Care Excellence. Clinical Knowledge Summaries. UK Medical Eligibility Criteria for use of copper intrauterine devices. 2011. http://www.cks.nhs.uk/ contraception_ius_iud/management/scenario_intrauterine_ device/starting_an_iud/uk_medical_eligibility_criteria [accessed 26 November 2012].

6 World Health Organization. Medical Eligibility Criteria for Contraceptive Use (4th edn). 2010. http://www.who.int/ reproductivehealth/publications/family_planning/ 9789241563888/en/ [accessed 26 November 2012].

7 Centers for Disease Control and Prevention. MMWR Early Release: U.S. Medical Eligibility Criteria for Contraceptive Use, Vol. 59. 2010. http://www.cdc.gov/mmwr/pdf/rr/rr59e0528.pdf [accessed 26 November 2012].

8 ACOG Committee on Practice Bulletins-Gynecology. Committee Opinion No. 539: Adolescents and long-acting reversible contraception: implants and intrauterine devices. Obstet Gynecol 2012;120:983-988.

9 Bahamondes MV, Hidalgo MM, Bahamondes L, et al. Ease of insertion and clinical performance of the levonorgestrel-releasing intrauterine system in nulligravidas. Contraception 2011;84: e11-e16.

10 Hubacher D, Reyes V, Lillo S, et al. Pain from copper intrauterine device insertion: randomized trial of prophylactic ibuprofen. Am J Obstet Gynecol 2006;195:1272-1277.

11 Lassner KJ, Chen CH, Kropsch LA, et al. Comparative study of safety and efficacy of IUD insertions by physicians and nursing personnel in Brazil. Bull Pan Am Health Organ 1995;29:206-215.

12 Chi IC, Galich LF, Tauber PF, et al. Severe pain at interval IUD insertion: a case-control analysis of patient risk factors. Contraception 1986;34:483-495.

13 Brockmeyer A, Kishen M, Webb A. Experience of IUD/IUS insertions and clinical performance in nulliparous women-a pilot study. Eur J Contracept Reprod Health Care 2008;13:248-254.

14 Marions L, Lövkvist L, Taube A, et al. Use of the levonorgestrel releasing-intrauterine system in nulliparous women - a non-interventional study in Sweden. Eur J Contracept Reprod Health Care 2011;16:126-134.

15 Heikinheimo O, Inki P, Kunz M, et al. Double-blind, randomized, placebo-controlled study on the effect of misoprostol on ease of consecutive insertion of the levonorgestrel-releasing intrauterine system. Contraception 2010;81:481-486.

16 Allen RH, Bartz D, Grimes DA, et al. Interventions for pain with intrauterine device insertion. Cochrane Database Syst Rev 2009;3:CD007373.

17 Fiala C, Gemzell-Danielsson K, Tang OS, et al. Cervical priming with misoprostol prior to transcervical procedures. Int J Gynaecol Obstet 2007;99(Suppl. 2):S168-S171.

18 Gemzell-Danielsson K, Mansour D, Fiala C, et al. Management of pain associated with the insertion of intrauterine contraceptives. Hum Reprod Update 2013;19:419-427.

19 Murty J. Use and effectiveness of oral analgesia when fitting an intrauterine device. J Fam Plann Reprod Health Care 2003;29:150-151.

20 Zhou L, Harrison-Woolrych M, Coulter DM. Use of the New Zealand Intensive Medicines Monitoring Programme to study 
the levonorgestrel-releasing intrauterine device (Mirena). Pharmacoepidemiol Drug Saf 2003;12:371-377.

21 Faúndes A, Alvarez F, Díaz J. A Latin American experience with levonorgestrel IUD. Ann Med 1993;25:149-153.

22 Sääv I, Aronsson A, Marions L, et al. Cervical priming with sublingual misoprostol prior to insertion of an intrauterine device in nulliparous women: a randomized controlled trial. Hum Reprod 2007;22:2647-2652.

23 Dijkhuizen K, Dekkers OM, Holleboom CA, et al. Vaginal misoprostol prior to insertion of an intrauterine device: an RCT. Hum Reprod 2011;26:323-329.

24 Gupta JK, Johnson N. Effect of mifepristone on dilatation of the pregnant and non-pregnant cervix. Lancet 1990;335: 1238-1240.
25 Faculty of Sexual and Reproductive Healthcare Clinical Effectiveness Unit. FSRH Guidance (Nov 2007) Intrauterine Contraception. 2007. http://www.fsrh.org/pdfs/ CEUGuidanceIntrauterineContraceptionNov07.pdf [accessed 14 March 2013].

26 Maguire K, Davis A, Rosario Tejeda L, et al. Intracervical lidocaine gel for intrauterine device insertion: a randomized clinical trial. Contraception 2012;86:214-219.

27 McNicholas CP, Madden T, Zhao Q, et al. Cervical lidocaine for IUD insertion pain: a randomized controlled trial. Am J Obstet Gynecol 2012;207:384.e1-e6.

28 Nadelberg R. Paracervical Block. Boston Scientific Corporation. 2007. http://www.bostonscientific.com [accessed 26 November 2012]. 